\title{
Impaired theory of mind in unaffected first-degree relatives of patients with anorexia nervosa
}

Tapajóz F. , Soneira S., Catoira N., Aulicino A., Allegri R.F.

DOI: http://doi.org/10.1002/erv.2701

\begin{abstract}
Objective: Previous studies have shown theory of mind (ToM) is affected in patients with anorexia nervosa (AN). There has also been growing interest in the study of endophenotypes in psychiatric disorders, since they allow better understanding of genetic mechanisms underlying different conditions, making them potential targets for future treatment. The goal of this study was to investigate whether ToM inefficiencies observed in patients with $A N$, are shared by unaffected first-degree relatives. Method: Performance on two ToM tasks (Reading the Mind in the Eyes and Faux Pas Test) were compared in 17 unaffected first-degree relatives of AN patients and in 17 healthy individuals matched for age and level of education. Depression, anxiety, obsessive compulsive, and eating disorder symptoms were also assessed and correlated with ToM and clinical/demographic variables. Results: Significant differences between groups were observed in all ToM tasks, with relatives of AN patients showing poorer performance. ToM assessment did not correlate with any clinical or demographic variable. Conclusions: The preliminary results of this study suggest unaffected first-degree relatives of AN patients display similar patterns of difficulty in ToM as reported previously for AN patients, supporting the hypothesis that ToM inefficiencies are a familial trait in this condition. (c) 2019 John Wiley \& Sons, Ltd and Eating Disorders Association.
\end{abstract}

\section{Keywords:}

anorexia nervosa, neuropsychology, social cognition, theory of mind, unaffected first-degree relatives 